\title{
Compromiso de la función pulmonar en pacientes con fibrosis pulmonar idiopática
}

\author{
MÓNICA GUTIÉRREZ N.*, NICOLÁS SÁNCHEZ D.*, MARÍA CAROLINA CUELLAR G.*, \\ MARÍA ANGÉLICA RODRÍGUEZ S.* y ÁLVARO UNDURRAGA P.**
}

Pulmonary function impairment in patients with idiopathic pulmonary fibrosis

Idiopathic pulmonary fibrosis (IPF) is the most frecuent of idiopathic interstitial pneumonias. The most sensitive lung function test for its diagnosis is a low DLCO register. Objective: To analyze IPF's clinical features and to establish its functional disability by DLCO at the time of diagnosis and also to correlate DLCO with 6-minute walked distance test (DR6), saturation pre and post DR6 and forced vital capacity (FVC). Methods: We studied retrospective data from 31 patients with $I P F$, analyzing personal and family history. We also determined pulmonary function parameters: DLCO (stratified in 4 groups: normal, mild, moderate and severe impairment), FVC, DR6 with pre and post oxygen saturation. Functional impairment according to DLCO was severe in 7 patients and moderate in 19 out of the 31 patients. Results: An inverse association was observed between DLCO impairment and oxygen saturation levels in rest, exercise and the difference between them $(p<$ 0.001). Conclusion: Oxygen saturation level in exercise and rest, as well as the difference between them, have a good correlation with DLCO for IPF diagnosis.

Key words: Idiophatic pulmonary fibrosis, diagnosis, DLCO.

\section{Resumen}

La fibrosis pulmonar idiopática (FPI) es la más común de las neumonías intersticiales idiopáticas. De los exámenes de función pulmonar, la DLCO es la más sensible para su diagnóstico. Objetivo: Revisar las características de los pacientes con FPI y determinar su compromiso funcional según DLCO. Correlacionar la DLCO con el test de caminata de 6 minutos (DR6), saturación pre y post DR6 y CVF. Método: Estudio retrospectivo de 31 pacientes con FPI, se analizó antecedentes clínicos, familiares e índices de función pulmonar: DLCO (estratificada en normal, disminución leve, moderada y severa), CVF, DR6 y $\mathrm{SaO}_{2}$ antes y después de DR6. Resultados: El compromiso funcional observado según DLCO fue grave en 7 pacientes y moderado en 19. Hubo asociación inversa entre gravedad según DLCO, saturación en reposo y en ejercicio y con la diferencia de saturación en reposo y ejercicio ( $p<0,001)$. Conclusión: La saturación de oxígeno en reposo y ejercicio, así como la diferencia entre ellas, tuvieron buena correlación con DLCO para el diagnóstico de FPI.

Palabras clave: Fibrosis pulmonar idiopática, diagnóstico, DLCO.

\section{Introducción}

La Fibrosis pulmonar idiopática (FPI) corresponde al tipo más común de neumonía intersticial idiopática, constituyendo el $60 \%$ de los casos aproximadamente ${ }^{1}$. De causa desconocida, el compromiso es limitado al pulmón e histológicamente caracterizada por neumonía intersticial usual (UIP) ${ }^{2}$. En relación a otras enfermedades intersticiales del pulmón tiene mal pronóstico, con una sobrevida media, desde el diagnóstico de la FPI, de 3 a 6 años ${ }^{1,3,4}$. Se ha establecido

* Unidad de Broncopulmonar y Laboratorio de Función Pulmonar, Clínica INDISA.

** Servicio de Medicina, Instituto Nacional del Tórax. 
que el género masculino y la edad avanzada se asocian a mayor mortalidad ${ }^{1}$.

Para realizar el diagnóstico confirmatorio de FPI, es necesario tener una biopsia pulmonar. Sin embargo, la clínica asociada a un patrón radiológico o tomográfico típico y alteraciones de la función pulmonar características pueden ser suficientes para establecer el diagnóstico en un número importante de casos ${ }^{2}$.

Desde el punto de vista de la función pulmonar, en esta patología, hay una alteración restrictiva (disminución de capacidad vital $(\mathrm{CV})$ con relación $\mathrm{VEF}_{1} / \mathrm{CVF}$ normal o aumentada) y alteración del intercambio gaseoso con aumento de la diferencia alvéolo-arterial de oxígeno en reposo o en ejercicio ${ }^{2,4}$. La espirometría y volúmenes pulmonares evalúan el compromiso restrictivo, mientras que los gases arteriales en reposo-ejercicio y la capacidad de difusión de monóxido de carbono (DLCO), evalúan el intercambio gaseoso. Los gases arteriales en reposo y ejercicio se afectan precozmente al igual que la DLCO, mientras que la espirometría lo hace más tardíamente ${ }^{1}$. Sin embargo, se debe considerar que en estados muy precoces de la enfermedad todos ellos pueden ser normales ${ }^{2}$.

El objetivo de este estudio es revisar las características clínicas de un grupo de pacientes con FPI y determinar el grado de compromiso funcional pulmonar al momento del diagnóstico según su DLCO. Se estudió también la correlación que existe entre DLCO, CVF y distancia recorrida en seis minutos (DR6) en relación a distancia recorrida y porcentaje de saturación de la hemoglobina antes y después de la prueba.

Además en un subgrupo de pacientes, se efectuó seguimiento funcional a los 6 meses usando las mismas evaluaciones.

\section{Pacientes y Métodos}

Estudio retrospectivo de 31 pacientes con diagnóstico de FPI registrados en la base de datos del laboratorio de función pulmonar de la clínica INDISA entre 2003 y 2006. El diagnóstico de FPI se basó en la biopsia y/o TAC de tórax. El diagnóstico de FPI fue establecido por TAC típico en el $80 \%$ de los casos, de éstos, un $45 \%$ tenía biopsia; $20 \%$ tenía TAC atípico y todos ellos tenían biopsia confirmatoria.

A partir de la base de datos, se confeccionó una planilla de registro con la identificación del paciente, sexo, edad, tabaquismo, síntomas de presentación, antecedentes familiares e índices de función pulmonar al momento del diagnóstico: CVF expresada en litros y como porcentaje del teórico, utilizándose los valores de referencia de Gutiérrez y cols ${ }^{7}$, DLCO corregida según hemoglobina, expresada como porcentaje de los valores de referencia de Roca y $\operatorname{cols}^{8}$, DR6 en metros recorridos ${ }^{9,10}$, expresada como porcentaje del teórico de los valores de referencia ${ }^{9}$ y saturación arterial de oxígeno $\left(\mathrm{SaO}_{2}\right)$, medida con oxímetro de pulso, al inicio y al término de la prueba.

Además, se realizó seguimiento del estudio funcional a los 6 meses, considerando los mismos parámetros que al momento de la evaluación inicial.

Se excluyeron pacientes con diagnóstico concomitante de EPOC, cáncer pulmonar, enfermedades intersticiales de causas conocidas y secundarias a mesenquimopatías.

El estudio funcional se realizó en un equipo MedGraphics modelo profiler/Dx. Todas las pruebas cumplían con las normas establecidas por la $\mathrm{ATS}^{6-9}$. La DLCO se efectuó por el método de respiración única (Single breath). Se empleó un oxímetro de pulso NONIN modelo 850 . Todas las pruebas funcionales fueron realizadas por el mismo profesional.

Se clasificó a los pacientes según el grado de compromiso funcional de la DLCO:

- Normal: sobre el límite inferior de normalidad (LIN).

- Leve: menor del LIN y mayor o igual a $65 \%$.

- Moderado: menor de 65\% y mayor o igual al $40 \%$.

- Severo: menor de $40 \%$.

Posteriormente se clasificó según la CVF en:

- Normal (sobre el LIN).

- Deterioro leve: menor del LIN y mayor o igual a $65 \%$.

- Moderado: menor de $65 \%$ y mayor o igual a $50 \%$.

- Avanzado: menor de 50\%.

Luego se correlacionó la CVF, la distancia recorrida en la caminata de 6 min y porcentaje de $\mathrm{SaO}_{2}$ en reposo y al finalizar el test, con las diferentes categorías de compromiso funcional según DLCO. Por último se analizó el cambio de la $\mathrm{SaO}_{2}$ en reposo y ejercicio entre los diferentes grupos según la DLCO.

En 18 pacientes (58\%) fue posible realizar un seguimiento de la función pulmonar a los 6 meses.

\section{Análisis estadístico}

Las variables continuas se expresan mediante promedio, desviación estándar (DE), medianas 
y límites; las variables categóricas mediante frecuencias absolutas y relativas. Para el análisis de asociación entre categorías de DLCO con $\mathrm{SaO}_{2}$ se utilizó la prueba de Kruskal Wallis, excluyendo de éste a un individuo con valores normales. Para definir entre qué parejas había diferencias, se utilizó la prueba de Wilcoxon para muestras independientes aplicando corrección de Bonferroni para el nivel de significación.

Para la correlación entre la distancia de caminata, DLCO, CVF y saturación de oxígeno se utilizó correlación de Spearman, utilizando los porcentajes sobre el teórico para DLCO y CVF. Para el análisis del cambio en la $\mathrm{SaO}_{2}$ en reposo y ejercicio entre los diferentes grupos según DLCO se utilizó la correlación de Spearman.

Para todos los efectos se consideró significativa un valor de alfa de 0,05 a dos colas.

\section{Resultados}

De los 31 pacientes con diagnóstico de FPI, 11 eran mujeres y 20 hombres. La mediana de edad fue de 65 años con un rango entre 38 y 88 años.

Diecisiete pacientes tuvieron antecedentes de

Tabla 1. Clasificación del grado de compromiso funcional de los pacientes según categorías de DLCO

\begin{tabular}{lcc}
\hline Categoría según DLCO & n & \% \\
\hline Normal & 1 & 3,2 \\
Disfunción & & \\
Leve & 4 & 12,9 \\
Moderada & 19 & 61,3 \\
Grave & 7 & 22,6 \\
Total & 31 & 100 \\
\hline
\end{tabular}

DLCO: capacidad de difusión del monóxido de carbono. tabaquismo y 10 de ellos habían fumado más de 20 paquetes-año; 5 pacientes tenían el antecedente familiar de fibrosis pulmonar. El principal síntoma fue la tos (67\%), seguido de disnea (58\%). Al examen físico destacaron crépitos (83\%) e hipocratismo digital (32\%).

La CVF promedio basal fue de $2.700 \mathrm{ml}$ (DE: $\pm 714,6)$ correspondiente a un $71,3 \%$ del teórico (DE: $\pm 17,1)$, la DLCO corregida promedio fue de $12,9 \mathrm{ml} / \mathrm{min} / \mathrm{mmHg}(\mathrm{DE}: \pm 4,3)$ con un valor de 50,8\% del teórico (DE: $\pm 14,9$ ); la DR6 promedio fue de 419 metros (DE: $\pm 65,9)$ que correspondió a un $90,7 \%$ del valor teórico (DE: $\pm 12,1)$. La saturación de oxígeno en reposo promedio fue $95 \%$ (DE: $\pm 2,1$ ), mientras que en ejercicio fue $87 \%$ (DE: $\pm 7,5$ ). Ningún paciente presentó desaturación en reposo.

El compromiso funcional según DLCO fue severo en 7 , moderado en 19 , leve en 4 y normal en 1 (Tabla 1). En las Tablas 2, 3 y 4 se muestran los diferentes grupos de gravedad según $\mathrm{DLCO}$, en relación a los diferentes parámetros funcionales expresados como porcentaje del teórico.

Al comparar el grado de compromiso funcional según DLCO, con los valores de CVF se observa que no existe correlación entre ambos índices (Tabla 5).

En la DR6, no hubo diferencia en los metros caminados entre el grupo moderado y grave (409 versus $406 \mathrm{~m}$ ). El grupo leve recorrió un promedio de $468 \mathrm{~m}$ (Tabla 6).

Se observó una asociación inversa entre gravedad según DLCO y saturación en reposo $(\mathrm{p}<$ $0,001)$ y post ejercicio $(\mathrm{p}<0,001)$ (Tabla 7 ; Figuras 1 y 2).

Al realizar el análisis de asociación entre las diferentes categorías de DLCO según $\mathrm{SaO}_{2}$ en reposo, encontramos diferencia significativa entre los grupos moderado y grave $(\mathrm{p}=0,003)$ y entre los grupos leve y grave $(\mathrm{p}=0,009)$, no así entre los grupos leve y moderado $(\mathrm{p}=0,933)$

Tabla 2. Índices de función pulmonar en pacientes con compromiso funcional leve según la DLCO

\begin{tabular}{ccccc}
\hline $\begin{array}{c}\text { DLCO } \\
\text { (\% teórico) }\end{array}$ & $\begin{array}{c}\text { CVF } \\
\text { (\% teórico) }\end{array}$ & $\begin{array}{c}\text { DR 6 } \\
\text { (metros) }\end{array}$ & $\begin{array}{c}\mathbf{S a O}_{2} \text { reposo } \\
\text { (\%) }\end{array}$ & $\begin{array}{c}\mathbf{S a O}_{2} \text { post ejercicio } \\
\text { (\%) }\end{array}$ \\
\hline 70 & 63 & 345 & 98 & 96 \\
70 & 63 & 363 & 97 & 95 \\
71 & 67 & 576 & 96 & 88 \\
72 & 78 & 453 & 97 & 95 \\
Media \pm DE & $73,5 \pm 10,4$ & $468,8 \pm 87,8$ & $96,2 \pm 0,9$ & $92,75 \pm 3,3$ \\
\hline
\end{tabular}

DR6 $=$ distancia recorrida en caminata de 6 minutos. 
M. GUTIÉRREZ N. et al.

Tabla 3. Índices de función pulmonar en pacientes con compromiso funcional moderado según la DLCO

\begin{tabular}{ccccc}
\hline $\begin{array}{c}\text { DLCO } \\
\text { (\% teórico) }\end{array}$ & $\begin{array}{c}\text { CVF } \\
\text { (\% teórico) }\end{array}$ & $\begin{array}{c}\text { Caminata } \\
\text { (metros) }\end{array}$ & $\begin{array}{c}\text { SaO }_{2} \text { reposo } \\
\text { (\%) }\end{array}$ & $\begin{array}{c}\text { SaO }_{2} \text { post ejercicio } \\
\text { (\%) }\end{array}$ \\
\hline 40 & 44 & 339 & 95 & 84 \\
40 & 64 & 341 & 98 & 99 \\
43 & 56 & 423 & 96 & 89 \\
44 & 57 & 348 & 89 & 76 \\
46 & 65 & 408 & 96 & 88 \\
46 & 78 & 378 & 97 & 95 \\
48 & 66 & 483 & 94 & 73 \\
48 & 63 & 540 & 96 & 89 \\
48 & 97 & 441 & 95 & 87 \\
50 & 86 & 342 & 96 & 89 \\
51 & 68 & 477 & 97 & 84 \\
52 & 61 & 357 & 93 & 93 \\
57 & 64 & 441 & 97 & 94 \\
59 & 80 & 369 & 98 & 95 \\
59 & 58 & 462 & 98 & 93 \\
63 & 106 & 357 & 96 & 96,7 \\
63 & 83 & 375 & 97 & 95 \\
64 & 67 & 483 & 96 & 95 \\
65 & 86 & 483 & 95 & 95 \\
\hline
\end{tabular}

Tabla 4. Índices de función pulmonar en pacientes con compromiso funcional grave según la DLCO

\begin{tabular}{ccccc}
\hline $\begin{array}{c}\text { DLCO } \\
\text { (\% teórico) }\end{array}$ & $\begin{array}{c}\text { CVF } \\
\text { (\% teórico) }\end{array}$ & $\begin{array}{c}\text { Caminata } \\
\text { (metros) }\end{array}$ & $\begin{array}{c}\mathbf{S a O}_{2} \text { reposo } \\
\text { (\%) }\end{array}$ & $\begin{array}{c}\mathbf{S a O}_{2} \text { post ejercicio } \\
\text { (\%) }\end{array}$ \\
\hline 28 & 50 & 321 & 92 & 80 \\
28 & 55 & 450 & 92 & 74 \\
32 & 57 & 393 & 94 & 82 \\
32 & 91 & 396 & 95 & 76 \\
33 & 106 & 402 & 94 & 78 \\
34 & 50 & 441 & 94 & 78 \\
34 & 74 & 462 & 94 & 85 \\
Media \pm DE & $69 \pm 22,1$ & $409,3 \pm 47,8$ & $93,5 \pm 1,1$ & $79 \pm 3,6$ \\
\hline
\end{tabular}

Tabla 5. Relación entre valores de CVF (\% teórico) y las distintas categorías de compromiso funcional según DLCO

\begin{tabular}{lc}
\hline $\begin{array}{l}\text { Compromiso funcional } \\
\text { según DLCO }\end{array}$ & CVF $(\% \pm$ DE) \\
\hline Leve & $73,5 \pm 10,4$ \\
Moderado & $69,8 \pm 15,1$ \\
Grave & $69,0 \pm 22,1$ \\
\hline
\end{tabular}

Correlación no fue significativa $(\mathrm{p}=0,577)$
Tabla 6. Relación entre la distancia recorrida en 6 minutos (DR6) y el grado de compromiso funcional según DLCO

\begin{tabular}{lc}
\hline $\begin{array}{l}\text { Compromiso funcional } \\
\text { según DLCO }\end{array}$ & $\begin{array}{c}\text { DR6 }(\mathbf{m}) \\
\text { Media } \pm \mathbf{D E}\end{array}$ \\
\hline Leve & $468,8 \pm 87,8$ \\
Moderado & $406,7 \pm 60,9$ \\
Grave & $409,3 \pm 47,8$ \\
\hline Correlación no fue significativa $(\mathrm{p}=0,288)$
\end{tabular}

Rev Chil Enf Respir 2008; 24: 279-285 
Tabla 7. Correlación de Spearman entre DLCO (\% teórico), con saturación de oxígeno en reposo y post ejercicio

\begin{tabular}{lcc}
\hline & Rho & p \\
\hline $\begin{array}{l}\text { Saturación de } \\
\text { oxígeno en reposo }\end{array}$ & 0,622 & $<0,001$ \\
$\begin{array}{l}\text { Saturación de } \\
\text { oxígeno post ejercicio }\end{array}$ & 0,686 & $<0,001$ \\
\hline
\end{tabular}

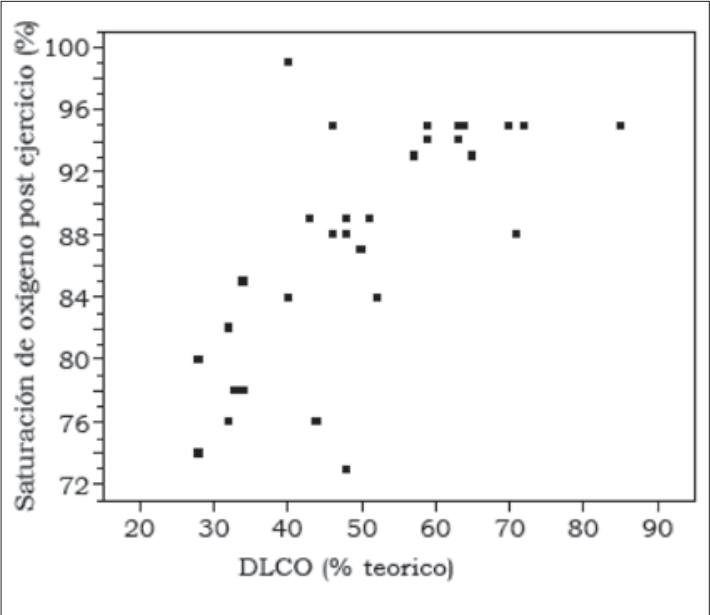

Figura 2. Gráfico de dispersión entre difusión de CO (\% teórico) y saturación de oxígeno post ejercicio.

En el análisis entre las diferentes categorías de DLCO y $\mathrm{SaO}_{2}$ post ejercicio, también se encontró una diferencia estadísticamente significativa entre los grupos moderado y grave $(p=0,003)$ $\mathrm{y}$ entre el grupo leve y grave $(\mathrm{p}=0,008)$. No se observó diferencia estadísticamente significativa entre el grupo leve y el moderado $(\mathrm{p}=0,433)$.

$\mathrm{Al}$ analizar la diferencia de $\mathrm{SaO}_{2}$ en reposo y ejercicio entre los diferentes grupos de DLCO, también se encontró una asociación inversa entre gravedad de DLCO y caída de la $\mathrm{SaO}_{2}(\mathrm{p}<0,001)$ (Figura 3).

Se logró realizar seguimiento funcional a los 6 meses en 18 pacientes (58\%). La mayoría se mantuvo en su grupo según DLCO, sólo un

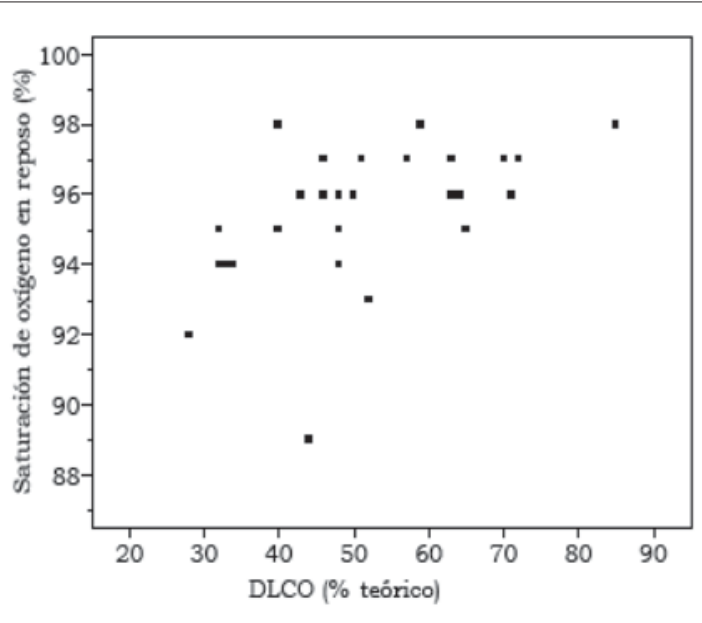

Figura 1. Gráfico de dispersión entre difusión de $\mathrm{CO}$ (\% teórico) y saturación de oxígeno en reposo.

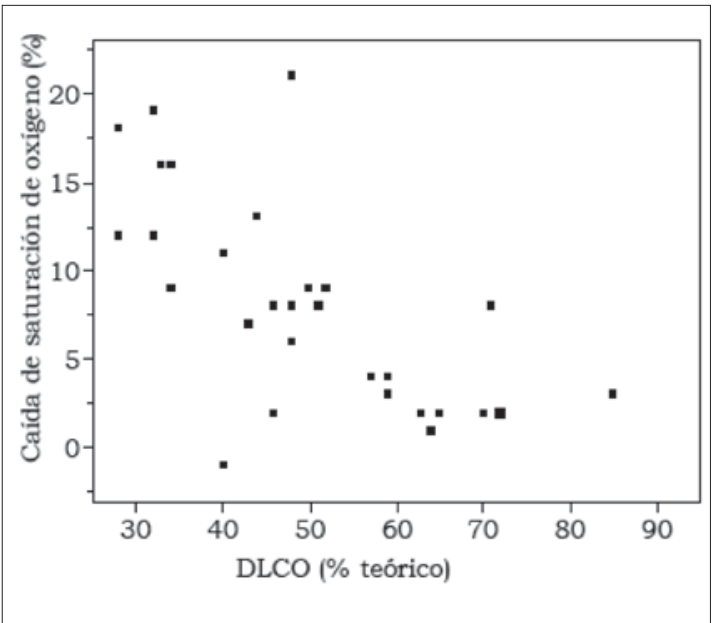

Figura 3. Gráfico de dispersión entre caída de la saturación y DLCO (\% teórico). Rho de Spearman -0,689 (p $<0,001)$.

paciente pasó del grupo moderado al leve y otro pasó del leve al moderado. Con respecto a las CVF, 3 pacientes presentaron una caída mayor al $10 \%$ en este período, sin relación con la severidad de la DLCO inicial. Los valores de DLCO en estos 3 pacientes no mostraron variaciones con respecto a los valores iniciales.

\section{Discusión}

En este estudio todos los pacientes tenían diagnóstico de FPI con alto grado de certeza basado en criterios tomográficos y/o anatomopatológicos. 
Se observó que la forma y edad de presentación de los pacientes concuerda con lo descrito previamente así como también los síntomas y el examen físico ${ }^{1-3}$. Sin embargo, el antecedente familiar presente en el $16 \%$ de los casos, es mayor que lo registrado en la literatura, en donde se describe como una condición inusual, en un rango que va desde 0,5 a $2,2 \%$, según datos de un estudio en el Reino Unido, que reporta una prevalencia de 1,34 casos por millón ${ }^{1,4}$. Otro estudio, reporta una FPI familiar de 3,3 a 3,7\% de los casos totales de $\mathrm{FPI}^{1,15}$. El mayor porcentaje de casos familiares de FPI obtenidos en este estudio, merece una investigación que aún no hemos emprendido.

Se agruparon los pacientes por gravedad según DLCO, ya que es la prueba funcional que ha demostrado ser más sensible para determinar grado de compromiso funcional y pronóstico en la FPI ${ }^{1,2,6}$. Se pudo observar, que al momento del diagnóstico, los pacientes catalogados como severos según su DLCO, presentaron valores de CVF normal o moderadamente alterados, lo que demuestra que en un paciente con sospecha de FPI que presenta CVF normal, no se puede descartar compromiso de la función pulmonar. En estos pacientes debe realizarse DLCO o una determinación de saturación antes y después del test de caminata. La CVF no parece ser un buen índice funcional para definir la extensión del daño pulmonar en la FPI, al momento del diagnóstico. Además, la coexistencia de enfisema pulmonar puede contrarrestar el efecto restrictivo de la fibrosis, resultando en una relativa preservación de los volúmenes pulmonares, pero marcada depresión de la DLCO alterando la relación entre volúmenes pulmonares y magnitud de la fibrosis ${ }^{1,5}$.

Pese a que el número de pacientes en este estudio es bajo, se encontró una asociación inversa estadísticamente significativa entre gravedad según valor de DLCO y caída de la saturación post ejercicio, definiendo caída significativa una variación de 4 puntos porcentuales ${ }^{16}$, según lo descrito en investigaciones previas ${ }^{6,16}$. El nivel de saturación menor a $88 \%$ al final del DR6 ha sido considerado, por diferentes autores, como índice de mal pronóstico, y ha sido asociado con la presencia de hipertensión pulmonar y alta mortalidad ${ }^{17-19}$, índices que no fueron determinados en este estudio.

En el análisis de seguimiento a 6 meses en un subgrupo de pacientes, no se observaron cambios significativos en valores de DLCO ni otros índices. Sin embargo, 3 pacientes presentaron una caída significativa de la CVF (definida como disminución mayor a 10\%). Las mediciones seriadas de la CV y CVF son aceptados como índices válidos que evalúan la progresión de la enfermedad, considerándose, la disminución mayor del $10 \%$ como índice de mal pronóstico ${ }^{1,2}$. De acuerdo a Martínez y cols ${ }^{20}$, sí se excluyen las exacerbaciones, la gran mayoría de los pacientes con FPI mantienen función pulmonar notablemente estable durante 6 meses de seguimiento.

Las limitaciones de este estudio están dadas por el pequeño número de casos y un tiempo de seguimiento aún insuficiente para validar los resultados obtenidos.

\section{Conclusiones}

- Los pacientes con FPI analizados en nuestro estudio, no difieren en características clínicas con lo ya descrito ${ }^{1}$.

- Los índices de función pulmonar analizados se comprometen heterogéneamente, siendo la DLCO el examen que demostró ser más precoz y sensible.

- El grado de alteración de la DLCO se relacionó bien con la desaturación post ejercicio, existiendo la mayor correlación entre el grupo con DLCO gravemente comprometido y la desaturación al final de la caminata de 6 minutos.

- A los 6 meses de seguimiento, la gran mayoría de los pacientes mantuvo una función pulmonar estable.

\section{Bibliografía}

1.- DEMPSEY O J. Clinical Review: Idiopathic pulmonary fibrosis-Past, present and future. Respiratory Medicine 2006; 100: 1871-85.

2.- AMERICAN THORACIC SOCIETY. Idiopathic pulmonary fibrosis: diagnosis and treatment. International consensus statement. American Thoracic Society (ATS) and the European Respiratory Society (ERS). Am J Respir Crit Care Med 2000; 161: 64664.

3.- GROSS T J, HUNNINGHAKE G W. Idiophatic Pulmonary Fibrosis. N Engl J Med 2001; 345: 517-25.

4.- MIKI K, MAEKUBA R, HIRAGA T. Impairments and prognosis factors for survival in patients with idiopathic pulmonary fibrosis. Respiratory Medicine 2003; 97: 482-90.

5.- DU BOIS R M, WELLS A U. Criptogenic fibrosing alveolitis/idiopathic pulmonary fibrosis. Eur Respir J 2001; 18 (suppl 32): 43-55.

6.- CHETTA A, MARANGIO E, OLIVIERI D. Pulmonary function testing in interstitial lung diseases. Respiration 2004; 71: 209-13.

7.- GUTIÉRREZ M, RIOSECO F, ROJAS A, CASANOVA 
D. Determinación de valores espirométricos en una población chilena normal mayor de 5 años, a nivel del mar. Rev Méd Chile 1996; 124: 1295-306.

8.- ROCA J, RODRÍGUEZ-ROISIN R, COBO E, BURGOS F, PÉREZ J, CLAUSEN JL. Single-breath carbon monoxide diffusing capacity prediction equations from a mediterranean population. Am Rev Respir Dis 1990; 141 (4 Pt 1): 1026-32.

9.- ENRIGHT P, SHERRILL D. Reference equations for the six-minute walk in healthy adults. Am J Respir Crit Care Med 1998; 158: 1384-7.

10.- ATS STATEMENT. Guidelines for the six-minute walk test. Am J Respir Crit Care Med 2002; 166: 111-7.

11.- Series ATS/ERS task force: Standarisation of lung function testing. General considerations for lung function testing. Eur Respir J 2005; 26: 153-61.

12.- SERIES ATS/ERS TASK FORCE. Standarisation of lung function testing. Standardisation of spirometry. Eur Respir J 2005; 26: 319-38.

13.- SERIES ATS/ERS TASK FORCE. Standarisation of lung function testing. Standardisation of Single-breath determination of carbon monoxide uptake in the lung. Eur Respir J 2005; 26: 720-35.

14.- MARSHALL R P, PUDDICOMBE A, COOKSON W
O, LAURENT G J. Adult familial cryptogenic fibrosis alveolitis in the United Kingdom. Thorax 2000; 55: 143-6.

15.- HODGSON U, LAITINEN T, TUKIAINEN P. Nationwide prevalence of sporadic and familial idiopathic pulmonary fibrosis: evidence of founder effect among multiplex families in Finland. Thorax 2002; 57: 338-42.

16.- DAL CORSO S, DUARTE S R, NEDER J A, et al. A step to assess exercise-related oxygen desaturation in interstitial lung disease. Eur Resp J 2007; 29: 330-6.

17.- NATHAN S D, SHLOBIN O A, AHMAD S, URBANEK S, BARNETT S D. Pulmonary hypertension and pulmonary function testing in idiopathic pulmonary fibrosis. Chest 2007; 131: 657-63.

18.- NOTH I, MARTÍNEZ F J. Recent advances in idiopathic pulmonary fibrosis. Chest 2007; 132: 637-50.

19.- FLAHERTY K R, ANDREI A C, MURRAY S. Prognostic value of changes in physiology and six minutewalk test. Am J Respir Crit Care Med 2006; 174: 803-9.

20.- MARTÍNEZ F J, SAFRIN S, WEYCKER D, STARKO $\mathrm{K} \mathrm{M}$, BRADFORD $\mathrm{W}$ Z, KING $\mathrm{T}$ E, et al, and IPF Study Group. The clinical course of patients with pulmonary fibrosis. Ann Intern Med 2005; 142: 963-7.
Correspondencia a:

Dra. Mónica Gutiérrez N.

E-mail:monicagunav@gmail.com 